\title{
Voice Pattern Recognition using CNN for Simple Command of Smart Toy
}

\author{
Kyung-Min Lee ${ }^{1}$, Jong-Bok Ahn ${ }^{1}$, Deok-In Kim² ${ }^{2}$ In-Young Kim² ${ }^{2}$, Chul-Won Park ${ }^{1}$ \\ ${ }^{1}$ Gangneung-Wonju National University \\ 150 Namwon-ro Heungeop-myeon, Wonju City, South Korea \\ point2529@naver.com; ajb30735@naver.com; cwpark1@gwnu.ac.kr \\ ${ }^{2}$ Urimalsoft \\ 1 Gangwondaehak-ro, Chuncheon City, South Korea \\ deokin.kim@urimalsoft.com; urimalsoft@urimalsoft.com
}

\section{Extended Abstract}

Smart toy means a plaything or other objects that the computerized units are related to its operation or the computers. Smart toys have begun to evolve with the spread of personal computers, and toy markets and technology are evolving as the needs of infants and the public grow. A new smart toy has been proposed to accommodate the advantages of electronic games. Recently, digital puzzles, electronic toys, and multimedia toys have been developed [1, 2].

On the other hand, as the platform of computers improves, interest in artificial intelligence increases after AlphaGo. Since the present toy is driven only by the operation for the limited operation, if a user learns to operate a voice command in a specific operation, it may be possible to program the operation in multiple commands. A new software education model is proposed as a smart toy based on voice learning [4]. For the study of overseas, NLP (Natural Language Processing), CNN (Convolutional Neural Network) for sentence classification and a convolutional neural network for modelling sentences have been proposed [5-7]. Recently, a novel method of text representation on HNN (Hybrid Neural Network) and a novel hierarchical convolutional neural network for question answering over paragraphs were presented [8].

Baefoo DIY Smart Toy is a new concept smart toy model that is being developed for use as software education educational materials and the smart toy of Urimalsoft in South Korea [3, 9]. The voice command smart toy system is composed of the mobile terminal such as a smartphone application or a voice secretary device that learns a user voice command, a smart toy AI server that analyses a voice command of the user and performs learning, smart toys.

The artificial neural network used for voice pattern learning is CNN models. CNN models have subsequently been shown to be effective for NLP and have achieved excellent results in semantic parsing, search query retrieval, sentence modelling, and other traditional NLP tasks [5-7]. The voice data is pre-processed and symbolized, and then an educational pattern is generated. After CNN processes through learning, it decodes it and sends it to the controller that performs the operation of the smart toy [9].

In this paper, we developed a simulator for voice pattern learning using CNN. This simulator enables simple learning and for performing simple commands of smart toy using CNN. The DNN algorithm of this simulator consists of CNN. Through the ML (Machine Learning) configure window of the simulator, number of repetitions, embedding size, convolution filter size, number of convolution channels channel, and learning rate can be set [9]. The training data was written in text file separated by a tap. On the horizontal axis, voice commands (114 from forward to backward movement) are inputted, and on the vertical axis, pre-processed data of voice commands (tagging morpheme and parts of speech by analysing morpheme) are inputted. We implemented the simulator by CNN using Python, which simulator learns user's voice command, so the performance evaluation of the voice test pattern was performed [10]. We found that the best results were obtained when the number of iterations, vector size, convolution filter size, number of channels, and learning rate were 500, 100, 3 to 5, 100, and 0.03 , respectively.

\section{Acknowledgements}

This work was supported by Ministry of SMEs and Startups (2017080146). 


\section{References}

[1] Y. M. Kim, “A Study on the Smart Toy Design for Young Children,” M.S. Dissertation, EHWA Womans Univ., Seoul, South Korea, 2004, pp. 1-73.

[2] K. U. Lee, Approach of computer activity for infants, ChangJi Press, pp. 1-329, 1995.

[3] I. Y. Kim, C. W. Park et al., "Development of voice learning system for voice learning smart toy," Ministry of SMEs and Startups, SME Technology Development, Project Proposal, pp. 1-39, 2017, 5.

[4] H. J. Kim, "Fundamentals of Natural Language Processing and Morphological Analysis," IT playground, p. 1, 2013, 10.

[5] Y. Kim, "Convolutional Neural Networks for Sentence Classification," Proceedings of the 2014 Conference on Empirical Methods in Natural Language Processing (EMNLP 2014), pp. 1746-1751, 2014.

[6] N. Kalchbrenner, E. Grefenstette, P. Blunson, "A Convolutional Neural Network for Modelling Sentences," Proceedings of the 52nd Annual Meeting of the Association for Computational Linguistics, pp. 655-665, 2014.

[7] A. Hassan, A. Mahmood, "A Novel Hierarchical Convolutional Neural Network for Question Answering over Paragraphs," IEEE Access, vol. 6, pp. 13949-13957, 2018.

[8] I. H. Witten, E. Frank, M. A. Hall, C. J. Pal, Data Mining, Practical Machine Learning Tools and Techniques, 4th ed. Elsevier, Morgan Kaufmann, pp. 1-656, 2017.

[9] C. W. Park, I. Y. Kim, et al., "Development of voice learning system for voice learning smart toy development," Ministry of SMEs and Startups, SME Technology Development, $1^{\text {st }}$ year report, pp. 1-135, 2017.

[10] G. Spacagna, D. Slater, V. Zocca, Python Deep Learning. Packt Press, pp. 1-368, 2017. 